\title{
A Case of Cardiac Calcified Amorphous Tumor Presenting with Concomitant ST-Elevation Myocardial Infarction and Occipital Stroke and a Brief Review of the Literature
}

\author{
Kyaw Kyaw, ${ }^{1}$ Htun Latt, ${ }^{2}$ Sammy San Myint Aung, ${ }^{1}$ and Chanwit Roongsritong ${ }^{1}$ \\ ${ }^{1}$ Institute for Heart and Vascular Health, Renown Regional Medical Center, 1500 E. 2nd St. No. 302, Reno, NV 89502, USA \\ ${ }^{2}$ Department of Internal Medicine, University of Nevada-Reno, School of Medicine, 1155 Mill St. No. W11, Reno, NV 89502, USA \\ Correspondence should be addressed to Kyaw Kyaw; drkyawkyawmd@gmail.com
}

Received 12 September 2017; Revised 9 November 2017; Accepted 27 November 2017; Published 24 December 2017

Academic Editor: Assad Movahed

Copyright (c) 2017 Kyaw Kyaw et al. This is an open access article distributed under the Creative Commons Attribution License, which permits unrestricted use, distribution, and reproduction in any medium, provided the original work is properly cited.

Cardiac calcified amorphous tumor (CAT) is an extremely rare benign intracavitary tumor of the heart. It may mimic other cardiac tumors and can present with signs or symptoms of systemic embolization. There are limited data regarding CAT in the literature. We report a case of a 68-year-old woman with a cardiac CAT and mitral annular calcification (MAC), who presented with acute ST-elevation myocardial infarction (STEMI) and occipital stroke. After extensive review of the literature, we believe that this case is possibly the first description of a cardiac CAT presenting with STEMI. The CAT was surgically removed, and the diagnosis was confirmed by histology. The patient tolerated the surgery and reported no events at 6-month follow-up.

\section{Introduction}

Cardiac calcified amorphous tumor (CAT) is a rare benign intracavitary tumor of the heart. Like other cardiac tumors, CAT may present with dyspnea, syncope, and pulmonary or systemic embolization and obstruction [1-4]. Herein, we are reporting a case of CAT with mitral annular calcification, who presented with acute ST-elevation myocardial infarction (STEMI) and occipital stroke. To the best of our knowledge, our case perhaps is the first description of a cardiac CAT presenting with STEMI.

\section{Case Presentation}

A 68-year-old woman with a 40-pack-year smoking history but no known medical problem was brought in by ambulance for sudden onset of persistent, severe left-sided chest pain without radiation. The patient also reported a new onset of blurred vision. She denied history of hypertension, dyslipidemia, diabetes mellitus, coronary artery disease (CAD), or chronic kidney disease or family history of premature CAD. Initial vital signs were normal with oxygen saturation of $98 \%$ on 2-liter supplemental oxygen via nasal cannula. Physical examination was benign, without murmur or abnormal heart sounds. There were no focal neurological deficits or signs of peripheral embolization noted. Initial electrocardiogram (EKG) showed 1-2 mm ST elevation in inferior leads (II, III, and aVF) with reciprocal ST depression in leads I and aVL (Figure 1). Oral aspirin and sublingual nitroglycerin were given immediately. Intravenous heparin was also initiated. Emergent coronary angiography showed an occlusion in one of the acute marginal branches of the right coronary artery and slow flow in the posterior descending artery. There was no other significant CAD (Figure 2). Left ventricular wall motion and systolic function were normal. Subsequent transthoracic echocardiogram (TTE) showed a $1.2 \times 1.2 \mathrm{~cm}$ mobile mass attached to the base of the posterior mitral valve leaflet on ventricular aspect (Figure 3). Of note, the mitral annulus also appeared rather echogenic. No thrombus was noted in left atrium or left ventricle during TTE or transesophageal echocardiogram (TEE). Troponin I was $1.7 \mathrm{ng} / \mathrm{ml}$ on arrival. It increased to $26 \mathrm{ng} / \mathrm{ml} 10$ hours later. Her workups, including complete 


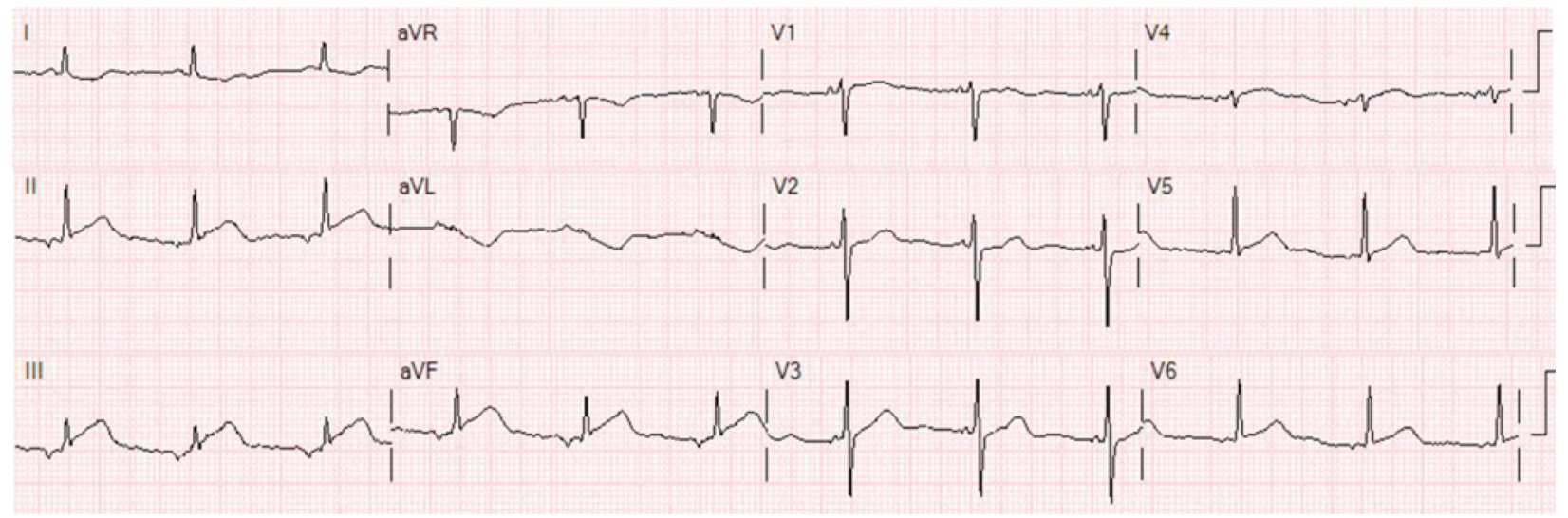

FIGURE 1: EKG showing ST elevation at II, III, and aVF with reciprocal ST depression at I and aVL.

blood count, prothrombin time/international normalized ratio, calcium, creatinine, liver function test, lipid panel, and hemoglobin A1c, were all normal. CT scan of the brain without contrast showed an infarct in the left occipital lobe (Figure 4). Cardiothoracic surgery was performed. A $1.2 \times 0.9 \times 0.2 \mathrm{~cm}$ tan-pink-colored soft tissue mass was surgically removed without any complications. Histologic examination of the mass showed a nodule of amorphous debris and fibrinous material with histiocytes and multinucleated giant cells without organism, consistent with a cardiac CAT (Figure 5). Throughout the hospital stay, she was closely monitored in coronary care unit and subsequently on telemetry floor. No atrial arrhythmia was reported.

The patient recovered well without recurrent symptoms at her 6-month follow-up.

\section{Discussion}

First introduced by Reynolds et al. in 1997, cardiac CAT is an extremely rare nonneoplastic primary tumor of the heart. It is characterized histologically by the features of calcified nodules and amorphous fibrinous materials [1]. CAT is usually discovered by cardiac imaging studies, but the definite diagnosis requires histological study of the tumor. ElBardissi et al. reported that the lifetime incidence of primary cardiac tumors (benign or malignant) was $0.02 \%$ based on 48 years of data collection, and only 8 of 323 cases $(2.47 \%)$ of primary cardiac tumors were CAT [5]. There have so far been limited data on pathophysiology, treatment, and prognosis of CAT. The majority of CAT is seen in association with valvular heart disease (31\%), end-stage renal disease $(21 \%)$, mitral annular calcification (MAC) (14\%), or diabetes mellitus (14\%) [2]. Of interest, the MACrelated CAT tends to be highly mobile and more likely to embolize [6]. To date, there have been 13 reported cases of MAC-related CAT [6-11]. Most of them (9 cases) are associated with ESRD. MAC, a chronic fibrous degeneration of the mitral valve, is reportedly caused by abnormalities of calcium-phosphorus $\left(\mathrm{Ca}-\mathrm{PO}_{4}\right)$ metabolism $[7-9,12]$. MAC has been reported to increase the incidence and mortality of cardiovascular diseases but has not been linked to increased

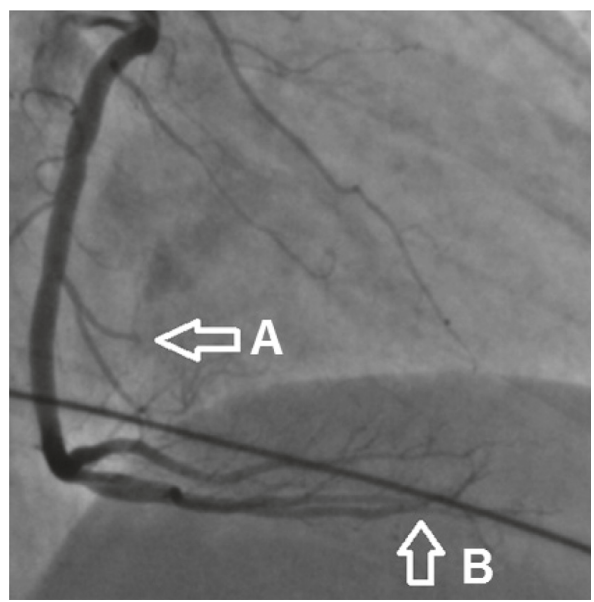

FIgURE 2: Coronary angiogram showing an occlusion of the small acute marginal branch (A) of the right coronary artery with slow flow in the posterior descending artery (B).

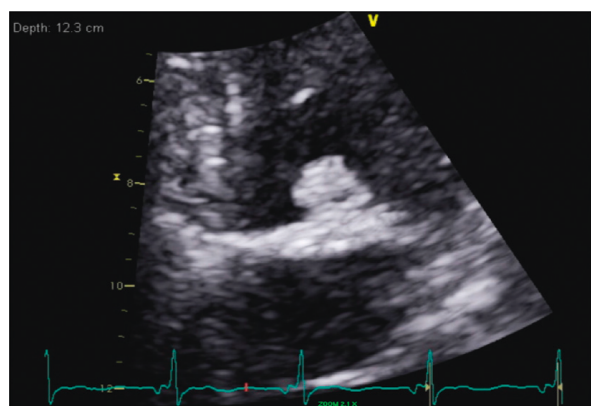

Figure 3: Transthoracic echocardiogram (TTE) showing a $1.2 \times 1.2 \mathrm{~cm}$ mobile mass attached to the ventricular aspect of mitral valve and hyperechogenic mitral annulus.

risk of myocardial infarction [13]. MAC is usually diagnosed based on hyperechoic mitral annulus on TTE. Our patient has normal renal function and serum calcium level. The echo densities of the tumor and mitral annulus in our patient are quite similar, suggesting the presence of MAClike appearance on TTE. We felt that the MAC-like appearance on TTE in our patient and other reported cases 


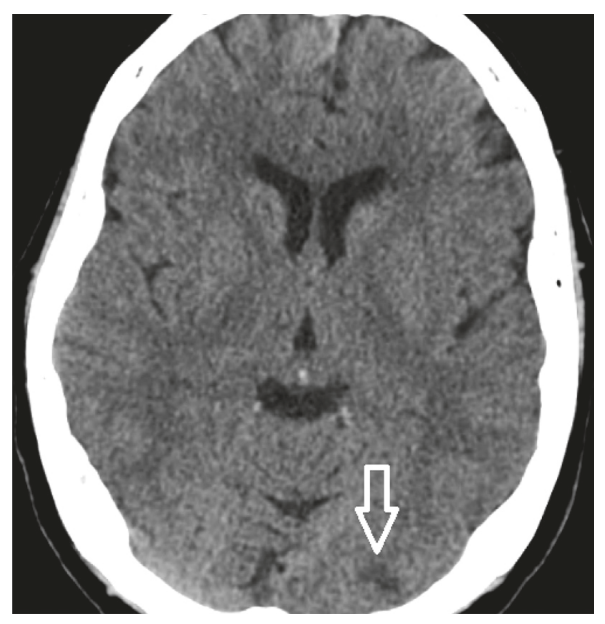

FIGURE 4: CT scan of the brain without contrast showing an infarct in the left occipital lobe.

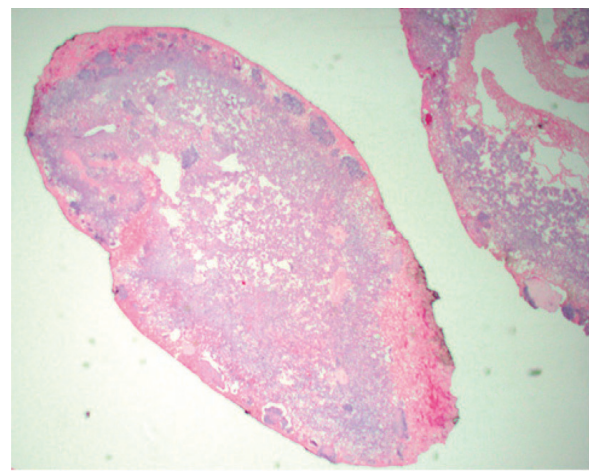

(a)

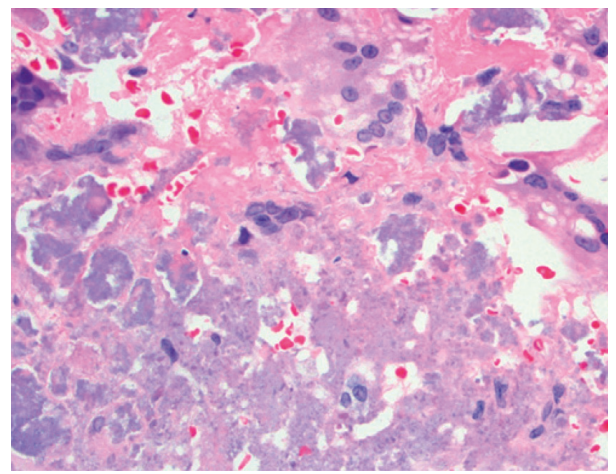

(b)

Figure 5: Pathology specimens showing a nodule of amorphous debris and fibrinous material in low-power field (a) and histiocytes and multinucleated giant cells in high-power field (b).

without associated ESRD may have resulted from tumor infiltration into mitral valve annulus rather than from the conventional MAC process due to abnormal Ca- $\mathrm{PO}_{4}$ metabolism. However, more studies are required to determine the exact underlying mechanism. Unfortunately, as in most patients who underwent surgical resection of the CAT, only the tumor was removed and biopsy of the mitral annulus was not performed in our patient. Surgical resection of the tumor is generally recommended to prevent embolization in most patients $[6,8-10,14]$. Our patient presented with concomitant blurred vision and STEMI, most likely due to the embolic phenomenon from the cardiac CAT.

The patient did not have any evidence of atrial arrhythmias on telemetry monitoring or intracardiac thrombus in TTE or TEE. Additionally, coronary angiogram showed isolated lesions at the third acute marginal branch and PDA of RCA and otherwise nonobstructive coronaries. Carotid ultrasound also showed normal bilateral vertebral and subclavian arteries, but there were mild stenoses $(<50 \%)$ of bilateral internal carotid arteries (ICAs). Mild
ICA stenoses could not explain the left occipital stroke as well. The occluded acute marginal branch of the right coronary artery was $<1.5 \mathrm{~mm}$ in diameter angiographically. Therefore, no coronary intervention or aspiration was performed. The prognosis is generally good after complete removal of the mass lesion $[15,16]$. The only recurrence was reported in a patient who received incomplete resection of the tumor [17]. Our patient tolerated the surgical procedure and has done well without any recurrent events at the sixmonth follow-up.

\section{Conclusion}

CAT of the heart is rare. Despite its benign nature, it can be highly mobile and consequently lead to systemic embolization, particularly in those with associated MAC. The prognosis is excellent with surgical resection in most patients. For those without ESRD or abnormal calcium metabolism, further studies may help determine whether cardiac CAT can involve the mitral annulus and result in an appearance similar to MAC on TTE. 


\section{Conflicts of Interest}

The authors declare that there are no conflicts of interest regarding the publication of this paper.

\section{References}

[1] C. Reynolds, H. D. Tazelaar, and W. D. Edwards, "Calcified amorphous tumor of the heart (cardiac CAT)," Human Pathology, vol. 28, no. 5, pp. 601-606, 1997.

[2] Q. de Hemptinne, D. De Cannière, J. L. Vandenbossche, and P. Unger, "Cardiac calcified amorphous tumor: a systematic review of the literature," IJC Heart \& Vasculature, vol. 7, pp. 1-5, 2015.

[3] N. Hussain, N. Rahman, and A. Rehman, "Calcified amorphous tumors (CATs) of the heart," Cardiovascular Pathology, vol. 23, no. 6, pp. 369-371, 2014.

[4] E. K. Choi, J. Y. Ro, and A. G. Ayala, "Calcified amorphous tumor of the heart: case report and review of the literature," Methodist DeBakey Cardiovascular Journal, vol. 10, no. 1, pp. 38-40, 2014.

[5] A. W. ElBardissi, J. A. Dearani, R. C. Daly et al., "Survival after resection of primary cardiac tumors," Circulation, vol. 118, no. 14, pp. S7-S15, 2008.

[6] Q. de Hemptinne, J .P. Bar, D. de Cannière, and P. Unger, "Swinging cardiac calcified amorphous tumour arising from a calcified mitral annulus in a patient with normal renal function," BMJ Case Reports, vol. 2015, p. bcr2014207401, 2015.

[7] T. Kawata, H. Konishi, A. Amano, and H. Daida, "Wavering calcified amorphous tumour of the heart in a haemodialysis patient," Interactive Cardiovascular and Thoracic Surgery, vol. 16, no. 2, pp. 219-220, 2012.

[8] S. Matsukuma, K. Eishi, K. Tanigawa et al., "Swinging calcified amorphous tumors with related mitral annular calcification," Annals of thoracic surgery, vol. 101, no. 4, pp. e103-e105, 2016.

[9] T. Takeuchi, K. Dohi, Y. Sato et al., "Calcified amorphous tumor of the heart in a hemodialysis patient," Echocardiography, vol. 33, no. 12, pp. 1926-1928, 2016.

[10] S. Katsuki, K. Ito, T. Tadokoro, T. Sasaguri, M. Hisahara, and M. Mohri, "Mitral annulus calcification-related calcified amorphous tumour trapped and extended by closing aortic valve," European Heart Journal-Cardiovascular Imaging, vol. 17, no. 5, p. 585, 2016.

[11] R. Nakamaru, H. Oe, K. Iwakura, T. Masai, and K. Fujii, "Calcified amorphous tumor of the heart with mitral annular calcification: a case report," Journal of Medical Case Reports, vol. 11, no. 1, p. 195, 2017.

[12] Y. Abramowitz, H. Jilaihawi, T. Chakravarty, M. J. Mack, and R. R. Makkar, "Mitral annulus calcification," Journal of the American College of Cardiology, vol. 66, no. 17, pp. 1934-1941, 2015.

[13] C. S. Fox, R. S. Vasan, H. Parise et al., "Mitral annular calcification predicts cardiovascular morbidity and mortality," Circulation, vol. 107, no. 11, pp. 1492-1496, 2003.

[14] M. AbbasiTeshnizi, A. Ghorbanzadeh, N. Zirak, B. Manafi, and A. Moeinipour, "Cardiac calcified amorphous tumor of the mitral valve presenting as transient ischemic attack," Case Reports in Cardiology, vol. 2017, Article ID 2376096, 3 pages, 2017.

[15] M. Kinoshita, H. Okayama, G. Kawamura et al., "A calcified amorphous tumor that developed on both sides of the atrioventricular valve annulus," Journal of Echocardiography, vol. 13, no. 4, pp. 148-150, 2015.
[16] F. Sabzi, H. Karim, B. Eizadi, R. Faraji, and N. Javid, "Calcified amorphous tumor of the heart with purple digit," Journal of Cardiovascular and Thoracic Research, vol. 6, no. 4, pp. 261264, 2014.

[17] M. E. Fealey, W. D. Edwards, C. A. Reynolds, P. A. Pellikka, and J. A. Dearani, "Recurrent cardiac calcific amorphous tumor: the CAT had a kitten," Cardiovascular Pathology, vol. 16, no. 2, pp. 115-118, 2007. 


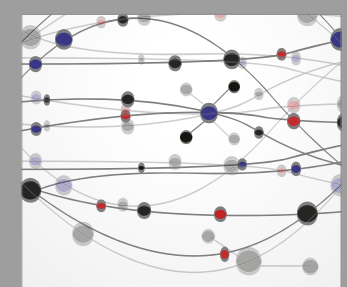

The Scientific World Journal
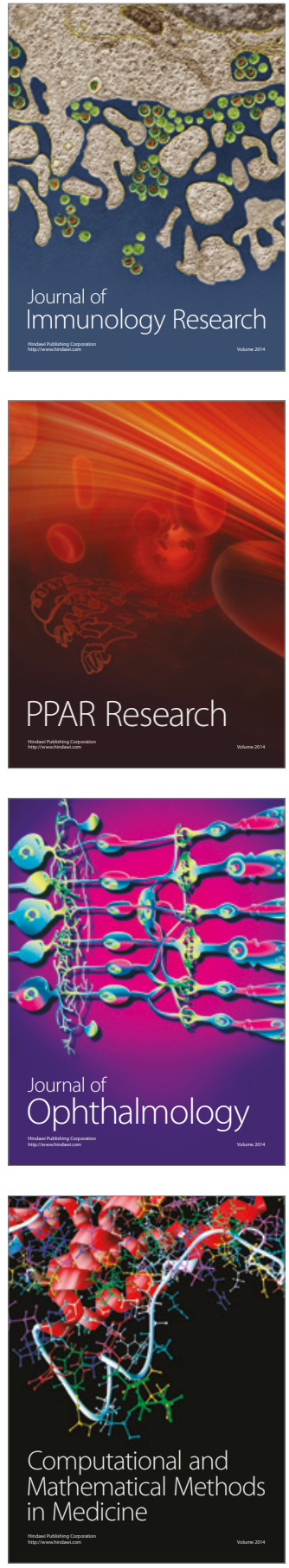

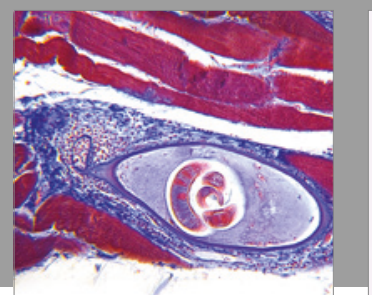

Gastroenterology Research and Practice
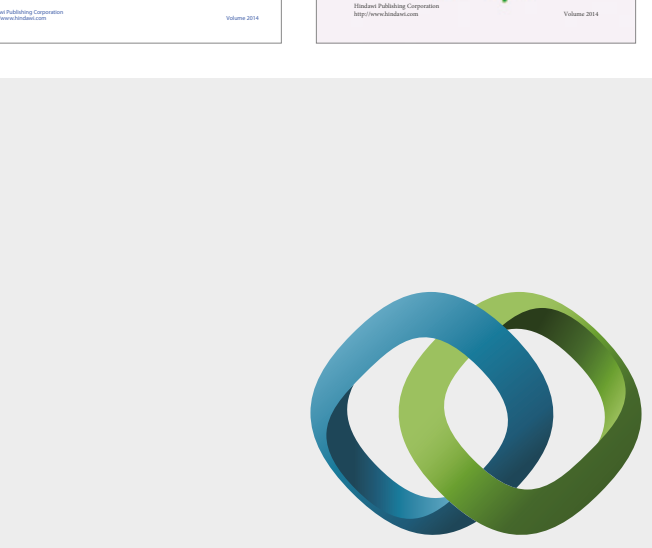

\section{Hindawi}

Submit your manuscripts at

https://www.hindawi.com
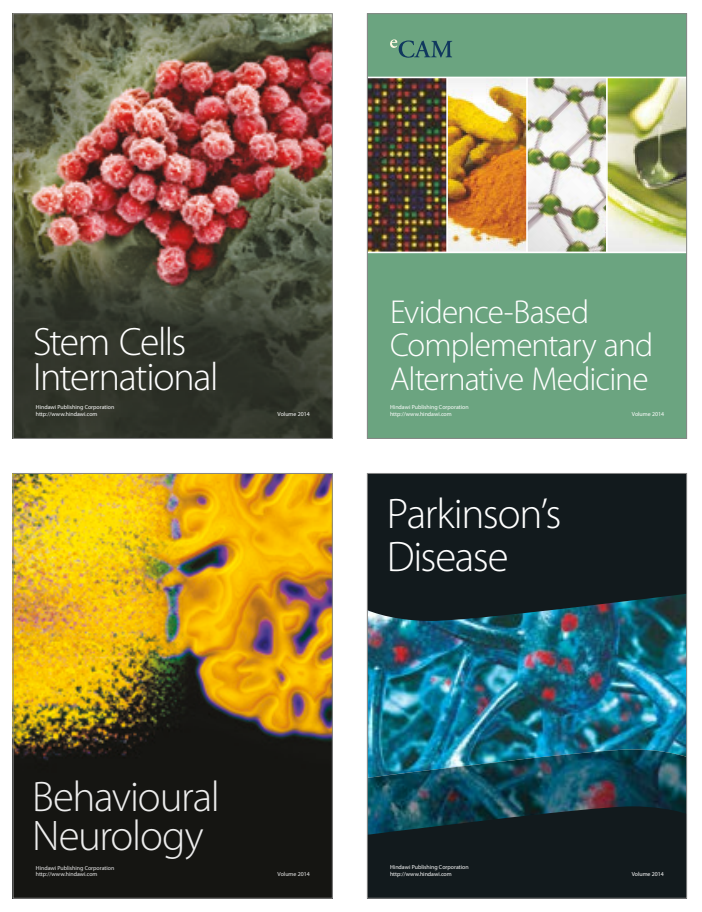
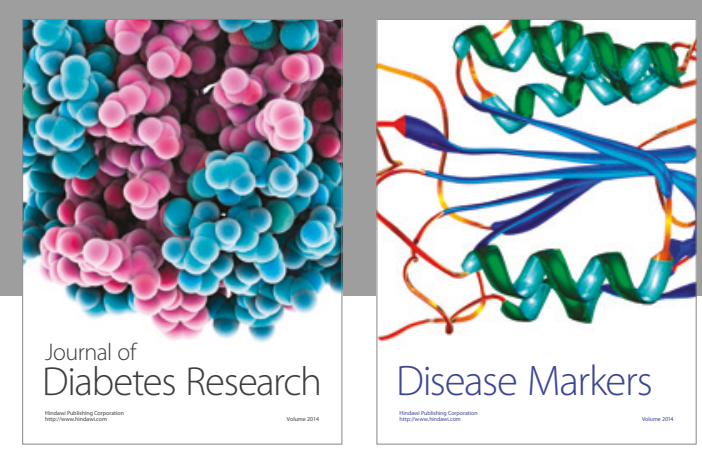

Disease Markers
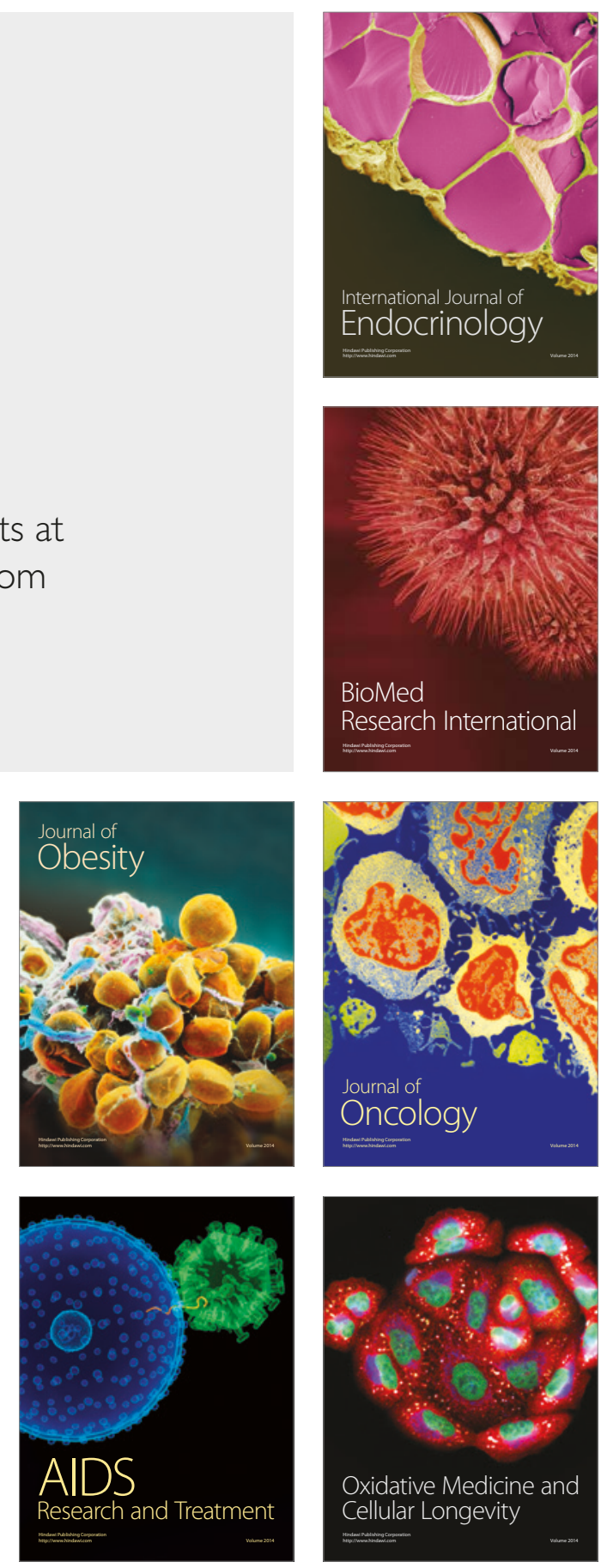